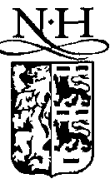

ELSEVIER

\title{
A generic, hard transition to chaos
}

\author{
O. López-Rebollal, J.R. Sanmartín \\ Depto. Física Aplicada. E.T.S. Ingenieros Aeronáuticos, Universidad Politécnica, 28040 Madrid, Spain
}

Received 23 March 1995; revised 9 June 1995; accepted 12 June 1995

Communicated by F.H. Busse

\begin{abstract}
A hard-in-amplitude transition to chaos in a class of dissipative flows of broad applicability is presented. For positive values of a parameter $\Gamma$, no matter how small, a fully developed chaotic attractor exists within some domain of additional parameters, whereas no chaotic behavior exists for $\Gamma \leq 0$. As $\Gamma$ is made positive, an unstable fixed point reaches an invariant plane to enter a phase half-space of physical solutions; the ghosts of a line of fixed points and a rich heteroclinic structure existing at $\Gamma=0$ make the limits $t \rightarrow+\infty, \Gamma \rightarrow+0$ non-commuting, and allow an exact description of the chaotic flow. The formal structure of flows that exhibit the transition is determined. A subclass of such flows (coupled oscillators in near-resonance at any $2: q$ frequency ratio, with $\Gamma$ representing linear excitation of the first oscillator) is fully analysed.
\end{abstract}

Keywords: Chaos; Maps; Oscillators; Resonances

\section{Introduction}

Deterministic chaos has a number of well-established results, mostly on discrete maps and, to a lesser extent, on flows. Typically, the route to chaos is marked by parameters running sequences of values that show bifurcation behavior. In the present work we report on a sudden (hard in amplitude) transition to chaos occurring in a class of systems described by dissipative flows. The transition exhibits both generic mathematical character and broad physical applicability, and allows deriving exact results.

For systems in the class, the $(t \rightarrow+\infty)$ attractor is a state of rest if a parameter $\Gamma$ is negative, and a simple set of fixed points and periodic orbits if $\Gamma$ vanishes. On the other hand, for $\Gamma$ positive, no matter how small, there is a fully developed chaotic attractor in certain domain of a space of additional parameters. Accordingly, the $\Gamma \rightarrow+0$ limit of long-time solutions to the flow is a singular solution: it does not exist at $\Gamma=0$ [1].

The non-commutability of limits $t \rightarrow+\infty, \Gamma \rightarrow+0$ relates to the fact that characteristic times diverge as $\Gamma \rightarrow+0$, the singular solution taking an infinite time to make a single leg on the attractor. As a consequence, the system may be described by an exactly one-dimensional (1D), noninvertible map; the Cantor structure that preserves determinism in passing from flows of dimension 3 and over to 1D maps is entirely washed off by the infinite time spent in each iteration. Since maps such as above are fully understood, one may consider the $\Gamma \rightarrow+0$ attractor as an exact representation of chaotic flow [2]. Note that the transition is soft in frequency. 
For a restricted subclass of systems exhibiting this transition, the phase-space topology is as follows. The flows have an invariant (plane) surface $\Sigma$. For $\Gamma<0$, a (saddle-node) fixed point $P$ with a 1D unstable manifold lies in a (nonphysical) half-space on one side of $\Sigma$, the system being attracted to rest on the other side. At $\Gamma=0, P$ reaches $\Sigma$, where it generates a line $\Lambda$ of fixed points and a second invariant surface $\Sigma^{\prime}$ that intersects $\Sigma$ at $\Lambda$. A family of heteroclinic orbits on $\Sigma$ joins $\Lambda$-points pairwise, ending in a homoclinic orbit at a point $Q$, with a family of periodic orbits beyond. Points on the arc $Q P$ of $\Lambda$ have no unstable manifold (their linearized flows have 1 zero, 2 negative eigenvalues; $P$ is doubly degenerate). A second family of heteroclinic orbits on $\Sigma^{\prime}$, nested at $P$, again joins $A$ points pairwise.

The entire heteroclinic (and periodic) structures are structurally unstable, disappearing with $A$ and $\Sigma^{\prime}$ at positive $\Gamma$; for $\Gamma$ small enough, $P$ is a saddle-focus with a 2D unstable manifold in the physical half-space, near $\Sigma$. For $\Gamma \rightarrow 0^{+}$, the spiraling motion lying between $P$ and $\Sigma$ is crashed onto $A$, making points on this line drift along it at vanishing rate. The $t \rightarrow+\infty, \Gamma \rightarrow+0$ motion is then made of pieces of $\Lambda$, together with $\Sigma$ and $\Sigma^{\prime}$ heteroclinic orbits. This allows the analytical determination of the exact 1D, bimodal map for a chaotic attractor, the extrema being related to both homoclinic point $Q$ and $A$-point paired to $P$ on $\Sigma$. There are systems that lack the invariant surface $\Sigma^{\prime}$ yet present this transition because, at $\Gamma=0, \Sigma$ is attracting; the chaotic attractor is then made of pieces of $\Lambda$, and of orbits that start from $\Lambda$-points and approach $\Sigma$-heteroclinic orbits in their return to $\Lambda$, and which may be numerically determined by two-way integration.

In Sections 2 and 3 we reconsider a classical problem ( $2: 1$ near-resonance of waves or oscillators with quadratic coupling), which is shown to exhibit the transition discussed; Hughes and Proctor [3] derived its 1D map at small $\Gamma$. Here, we describe in detail the phase-space topological features (first, for conservative coupling, Section 2) that lead to the sudden set up of a chaotic attractor. In Section 4 we determine the formal structure of the class of flows that exhibit this same hard transition. In Section 5 we analyse variants of the transition in $1: 1$ resonances. Results are summarized in Section 6.

\section{Conservative quadratic coupling}

In this section we retake an old problem in nonlinear conservative coupling. Our purpose is to point out the rich topology of a particular resonance. First, we review two well-established models for that resonance.

\subsection{Reduced 3-wave interaction}

The resonant interaction of nearly linear waves has been a subject of considerable interest for over 30 years, particularly in plasma physics [4,5]. The lowest order nonlinear effect is the 3-wave quadratic coupling, extensively reviewed by Kaup et al. [6]; for some recent results see Chow et al. [7]. This coupling is present in (a variety of) plasma waves, Rossby waves in the atmosphere, gravity-capillary waves in deep water, and both parametric amplifiers and nonlinear optics applications. Simultaneous quadratic and (non-conservative) cubic coupling has been considered by Guckenheimer and Mahalov [8].

For a lossless medium, the well-known equations for normal-mode complex amplitude $a_{j}, j=1-3$, in the space-independent case, are [5]

$$
\begin{aligned}
& \frac{\partial a_{1}}{\partial t}-i \omega_{1} a_{1}=-a_{2} a_{3} \\
& \frac{\partial a_{2}}{\partial t}-i \omega_{2} a_{2}=a_{3}^{*} a_{1}
\end{aligned}
$$




$$
\frac{\partial a_{3}}{\partial t}-i \omega_{3} a_{3}=a_{1} a_{2}^{*}
$$

The conveniently normalized amplitudes, and any frequency mismatch $\nu \equiv \omega_{1}-\omega_{2}-\omega_{3}$, are assumed small $\left(a_{j}, \nu \ll \omega_{j}\right)$, the quadratic terms in (1)-(3) making weak corrections to the linear mode frequencies $\omega_{j}$. For media off thermodynamic equilibrium, some waves might have negative energy; this would result in a plus sign in the right-hand-side of (1), leading to explosive behavior (all three amplitudes diverging at a finite time), a case here excluded [6].

Writing $a_{j} \rightarrow e^{i \omega_{j} t} \times a_{j} e^{i \phi_{j}}, a_{j}$ and $\phi_{j}$ real and slowly time dependent, leads to a fourth-order system of equations describing the slow evolution of $a_{1}, a_{2}, a_{3}$ and $\beta \equiv \frac{1}{2} \pi+\nu t+\phi_{1}-\phi_{2}-\phi_{3}$. We will not pause to write that system [5], just noting, for our purposes, that it admits three first integrals,

$$
\begin{aligned}
& a_{3}^{2}-a_{2}^{2}=\text { constant } \equiv K \\
& a_{1}^{2}+a_{2}^{2}=\text { constant } \equiv K^{\prime} \\
& \omega_{1} a_{1}^{2}+\omega_{2} a_{2}^{2}+\omega_{3} a_{3}^{2}-2 a_{1} a_{2} a_{3} \cos \beta=\text { constant } \equiv E
\end{aligned}
$$

The full solution, first obtained by Jurkus and Robson in 1960 [9], involves Jacobi elliptic functions. Here, we will be interested in the particular case $K=0$ (reduced 3-wave interaction, $a_{3} \equiv a_{2}$ ). The resulting equations for $a_{1}, a_{2}$ and $\beta$ are then

$$
\begin{aligned}
& \dot{a}_{1}=-a_{2}^{2} \sin \beta \\
& \dot{a}_{2}=a_{1} a_{2} \sin \beta, \\
& \dot{\beta}=\nu+2 a_{1} \cos \beta-\frac{a_{2}^{2}}{a_{1}} \cos \beta
\end{aligned}
$$

which admit integrals $K^{\prime}$ and $E$ (with $a_{3}=a_{2}$ ).

Resonant quadratic coupling may involve just two waves. Equations isomorphic to (7)-(9) were first derived by Armstrong et al. [10] for the interaction of a fundamental wave and its second harmonic in crystals. For harmonic generation in nonlinear optics the space-coordinate along a wave-guide took the place of time in the equations.

\subsection{Quadratic coupling of two oscillators}

The resonant interaction of oscillators with frequency ratio $2: 1$ was first studied by Mandelstam's collaborators in the early 30's. They considered vibrations within $\mathrm{CO}_{2}$ molecules, following a model for Raman scattering suggested by Fermi. Additional 2:1 resonance phenomena can be found in betatron beams, celestial mechanics, and the simple elastic pendulum, which was analysed in detail by Breitenberger and Mueller [11], where numerous references can be found.

Let $V(x, y)$ be a general two-dimensional potential energy, with normal coordinates $x, y$ and with the origin at a stable equilibrium point, and write

$$
\left.\frac{\partial^{2} V}{\partial x^{2}}\right|_{0,0} \equiv V_{x x}^{0} \equiv 4 m \omega_{0}^{2}, \quad V_{y y}^{0} \equiv m\left(\omega_{0}+\frac{1}{2} \nu\right)^{2}
$$

with $\nu / \omega_{0}$ small. Expanding $V$ up to terms cubic in $x, y$, the equations for a particle of mass $m$ moving near the origin are

$$
\ddot{x}+4 \omega_{0}^{2} x \simeq \frac{-1}{2 m}\left(V_{x x x}^{0} x^{2}+2 V_{x x y}^{0} x y+V_{x y y}^{0} y^{2}\right)
$$




$$
\ddot{y}+\omega_{0}^{2} y \simeq-\omega_{0} \nu y-\frac{1}{2 m}\left(V_{x x y}^{0} x^{2}+2 V_{x y y}^{0} x y+V_{y y y}^{0} y^{2}\right)
$$

Clearly, the $\nu$ and $V_{x y y}^{0}$ terms in the right-hand-sides of (10),(11) will result in slowly evolving amplitudes and phase "constants" for otherwise harmonic oscillations of $x$ and $y$ at frequencies $2 \omega_{0}$ and $\omega_{0}$, respectively. Writing

$$
x=\left(4 m \omega_{0} / V_{x y y}^{0}\right) a_{1} \cos \left(2 \omega_{0} t+\psi\right), \quad y=\left(8 m \omega_{0} / V_{x y y}^{0}\right) a_{2} \cos \left(\omega_{0} t+\theta\right)
$$

and averaging over the $2 \omega_{0}$ and $\omega_{0}$ periods recover Eqs. (7) $-(9)$ for $a_{1}, a_{2}$ and $\beta \equiv 2 \theta-\psi$.

Since phases $\theta, \psi$ enter system (7)-(9) through the combination $2 \theta-\psi$ only, there exists a cyclic angular coordinate, $K^{\prime}$ being the first integral for its action, resulting from the adiabatic character of changes in $x$ and $y$ oscillations [11]. The energy $E$, with $a_{3}=a_{2}$, includes linear wave and interaction contributions, and may be used as a Hamiltonian to derive (7)-(9) [5]. For the full 3-wave case, there are two cyclic phases and two corresponding first integrals, $K$ and $K^{\prime}$, in addition to the energy $E$; Eqs. (4),(5) are the Manley-Rowe relations for conservation of action densities in wave packets that follow particle Hamiltonian dynamics [12].

\subsection{Phase-space topology}

In discussing the chaotic flow of next section, it proves convenient to first elicit some particular topological features of the conservative system (7)-(9). From Eqs. (5),(6), with $a_{3}=a_{2}$, one gets a new family of invariant surfaces,

$$
a_{2}^{2}\left(\nu+2 a_{1} \cos \beta\right)=\mathrm{constant}=\omega_{1} K^{\prime}-E
$$

Consider the degenerate case $\omega_{1} K^{\prime}-E=0$, which is the product of invariant surfaces $\Sigma\left(a_{2}=0\right)$ and $\Sigma^{\prime}\left(\nu+2 a_{1} \cos \beta=0\right.$ ) (we will assume $\nu>0$; otherwise, write $\nu \rightarrow-\nu, \beta \rightarrow \pi-\beta$ in (7)-(9)). The intersection of both surfaces is a line $A$ of fixed points.

For $a_{2}=0$, Eqs. (7),(9) give

$$
a_{1}=\text { constant } \equiv a_{10}, \quad \dot{\beta}=\nu+2 a_{10} \cos \beta
$$

There are then three types of orbits in $\Sigma$, shown in Fig. 1 together with the line $A$. There is a homoclinic orbit through point $Q$, for $2 a_{10}=\nu$; a family of periodic orbits, with period $2 \pi /\left(\nu^{2}-4 a_{10}^{2}\right)^{1 / 2}$, for $2 a_{10}<\nu$; and a (double) family of heteroclinic orbits, joining points on opposite branches of $\Lambda$, for $2 a_{10}>\nu$. The corresponding solutions $\beta(t)$ are

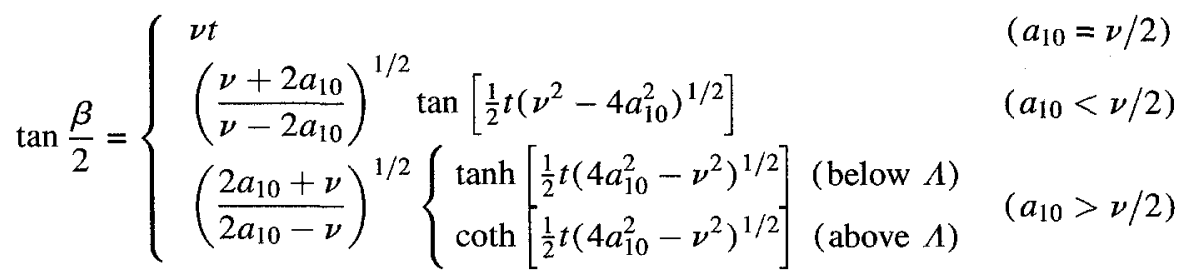

Taking $a_{10}$ as parameter, the entire structure is the result of a local (saddle-node) bifurcation at $Q$ ( $a_{10}=$ $\nu / 2, \beta=\pi$ ), together with a global topological feature (the $a_{1}-\beta$ manifold is a cylindrical surface, $\beta=0$ and $\beta=2 \pi$ representing the same states).

In the surface $\Sigma^{\prime}\left(\nu+2 a_{1} \cos \beta=0\right)$ there is another family of heteroclinic orbits again joining points in opposite branches of $A$ (Fig. 2). Eq. (9) now takes the form 


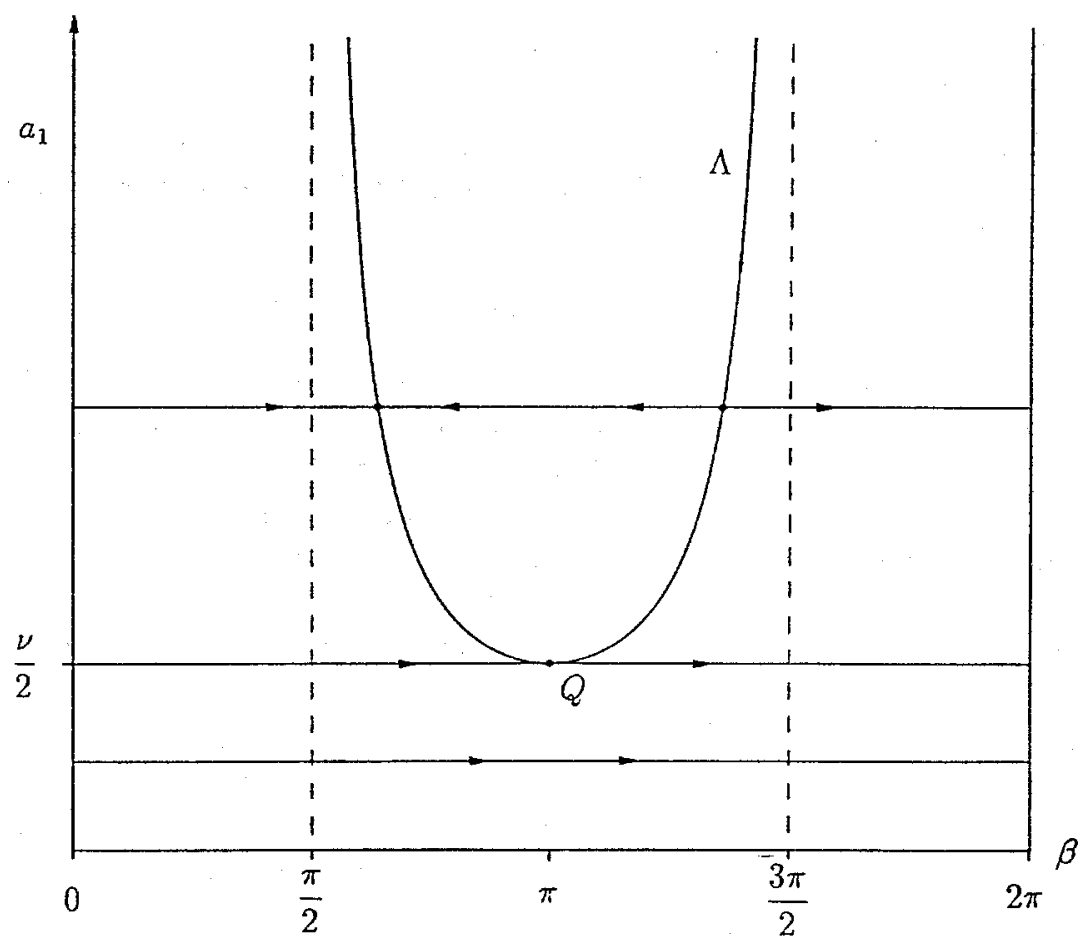

Fig. 1. Plane $a_{1}-\beta$ showing the line $A$ of fixed points, the homoclinic orbit through point $Q$, and periodic and heteroclinic orbits below and above it, respectively, for conservative $2: 1$ resonance.

$$
\dot{\beta}=-\frac{a_{2}^{2}}{a_{1}} \cos \beta
$$

and writing Eq. (5) as $a_{2}^{2}=a_{1 \infty}^{2}-a_{1}^{2}$ one finds

$$
\begin{aligned}
\tan \beta & =\frac{\left(4 a_{1 \infty}^{2}-\nu^{2}\right)^{1 / 2}}{\nu} \tanh \left[\frac{1}{2} t\left(4 a_{1 \infty}^{2}-\nu^{2}\right)^{1 / 2}\right] \\
& \equiv \tan \beta_{\infty} \times \tanh \left(\frac{1}{2} \nu t \tan \beta_{\infty}\right)
\end{aligned}
$$

All equilibria on the line $\Lambda$ are, naturally, degenerate. As shown by the above heteroclinic solutions $\beta(t)$, and as indicated in Figs. 1,2, the unstable (stable) manifold for points in the $\beta<\pi$ branch lies on the surface $\Sigma^{\prime}(\Sigma)$; the opposite holds for the branch $\beta>\pi$.

\section{Hard transition to chaos for quadratic coupling}

\subsection{Non-conservative coupling}

We are now in a position to consider 3-wave coupling with wave 1 linearly unstable and waves 2 and 3 equally damped. Adding terms $-\Gamma a_{1}, \gamma a_{2}$ and $\gamma a_{3}$ to the left of Eqs. (1),(2),(3), respectively, and writing $a_{j} \rightarrow e^{i \omega_{j} t} \times a_{j} e^{i \phi_{j}}, a_{3}^{2}-a_{2}^{2}$ is found to decay exponentially. There is, therefore, no loss of generality in setting $a_{3}=a_{2}$ from the outset in discussing attractors. Instead of Eqs. (7)-(9) one then gets

$$
\dot{a}_{1}=\Gamma a_{1}-a_{2}^{2} \sin \beta
$$




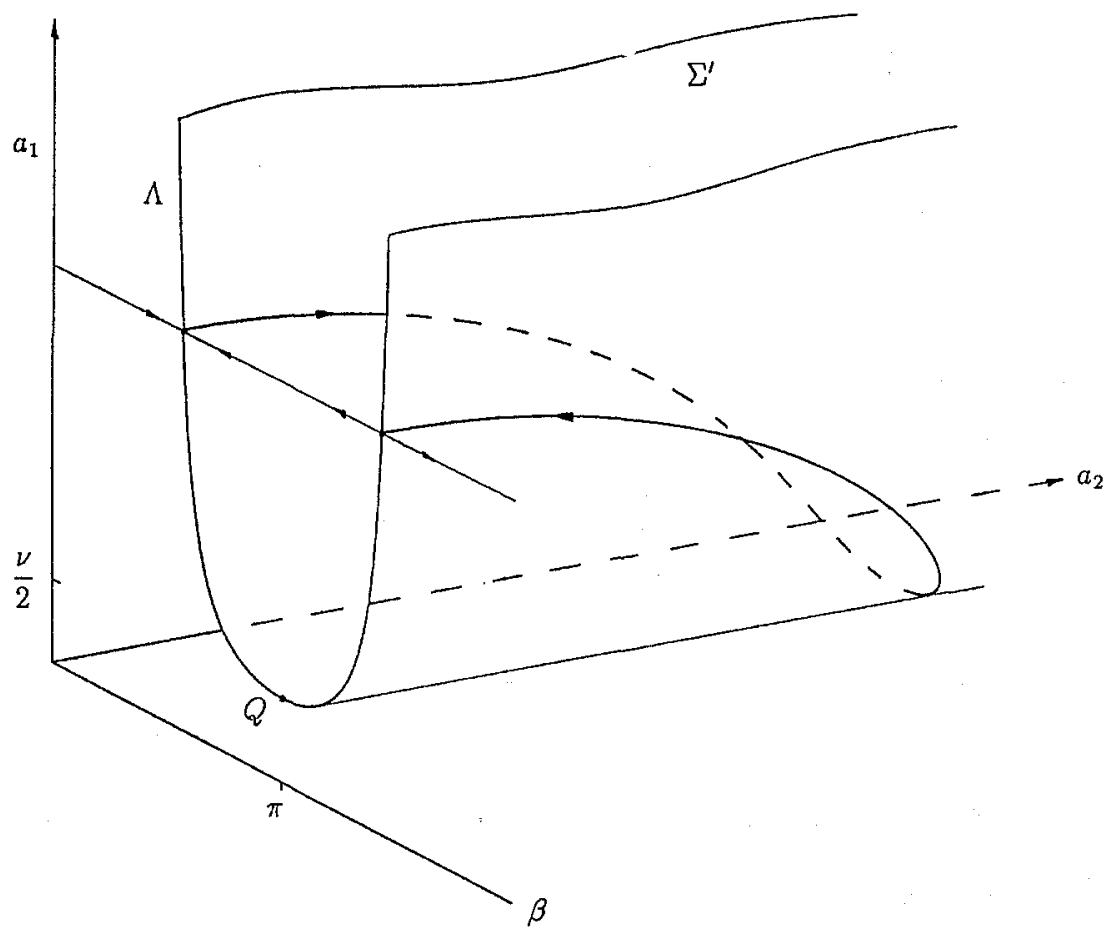

Fig. 2. Heteroclinic orbit contained in the invariant surface $\Sigma^{\prime}$, beginning and ending at two symmetric fixed points on the line $A$ of Fig. 1 .

$$
\begin{aligned}
& \dot{a}_{2}=-\gamma a_{2}+a_{1} a_{2} \sin \beta \\
& \dot{\beta}=\nu+2 a_{1} \cos \beta-\frac{a_{2}^{2}}{a_{1}} \cos \beta
\end{aligned}
$$

These equations have been extensively studied, using $a_{1} \cos \beta, a_{2} \cos \beta$ and $a_{2}^{2}$, or $\beta,\left(a_{1}^{2}+a_{2}^{2}\right)^{1 / 2}$ and $a_{2}^{2} /\left(a_{1}^{2}+a_{2}^{2}\right)$, as independent variables too [13-18,3].

System (14)-(16) also represents the coupling of an unstable mode and its damped first harmonic, or the interaction of two oscillators as described by Eqs. (10),(11), with terms $-2 \Gamma \dot{x}$ and $2 \gamma \dot{y}$ added to the respective left-hand-sides to represent net driving of the high-frequency $x$-oscillator, both of them being naturally damped. A physical variant of this last model requires no frequency mismatch $\nu$, a defective external feedback driving the $x$-oscillation at a constant phase mismatch $\sigma$ to the velocity response $\dot{x}$ [19] (see Section 5): to the left-hand-side of Eq. (10), one would add a damping term $2 \gamma \dot{x}$ with $\dot{x} \simeq-\left(8 m \omega_{0}^{2} / V_{x y y}^{0}\right) a_{1} \sin \left(2 \omega_{0} t+\psi\right)$, and a driving term $2 \Delta\left(8 m \omega_{0}^{2} / V_{x y y}^{0}\right) a_{1} \sin \left(2 \omega_{0} t+\psi-\sigma\right)$. Then, parameters $\Delta \cos \sigma-\gamma$ and $\Delta \sin \sigma$ would replace $\Gamma$ and $\nu$ in Eqs. (14),(16), respectively; since $\psi$ changes in times long compared with $\omega_{0}^{-1}$, the mismatch $\sigma$ could just result from a drive $-2 \Delta \dot{x}(t-\tau)$ that lags the response by the time $\tau=\sigma / 2 \omega_{0}$.

We first briefly sum up simple, known results derived analytically from (14)-(16). The flow divergence, arising solely from the linear, non-Hamiltonian terms,

$$
\frac{1}{a_{1}} \frac{\partial}{\partial a_{1}} a_{1} \dot{a}_{1}+\frac{1}{a_{2}} \frac{\partial}{\partial a_{2}} a_{2} \dot{a}_{2}+\frac{\partial \dot{\beta}}{\partial \beta}=2(\Gamma-\gamma)
$$

remains negative (and constant throughout phase-space) as long as $\Gamma<\gamma$. If both amplitudes are damped ( $\Gamma<0$ ) the state of rest, $a_{1}=a_{2}=0$, is a global attractor. For $\Gamma>0$, however, the equilibrium $a_{1}=a_{2}=0$ is unstable; there is now a second fixed point $P$ (Fig. 3 ), 


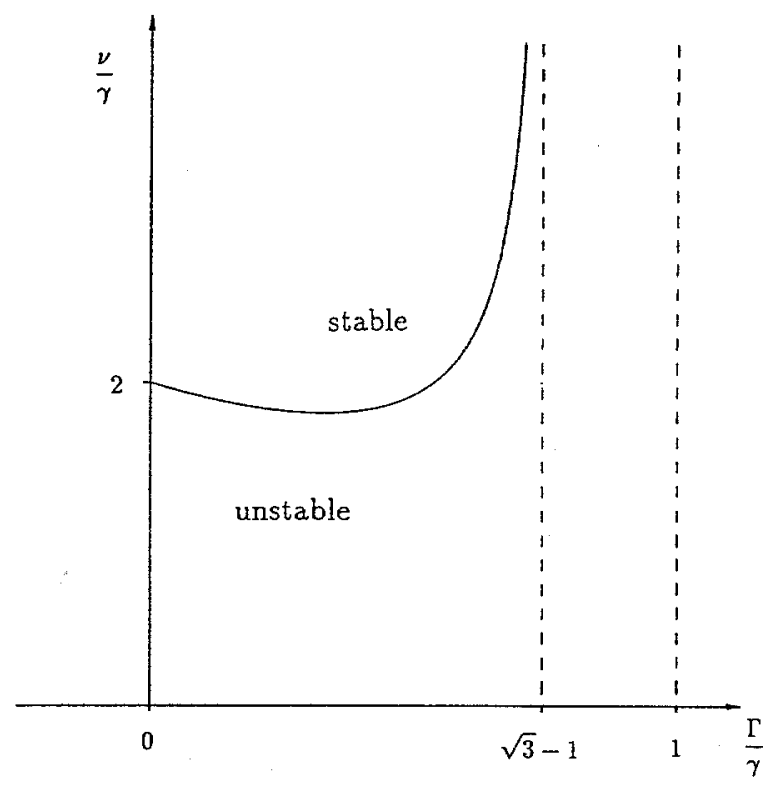

Fig. 3. Plane ( $\nu \gamma, \Gamma \gamma)$ showing the stability boundary (Eq. (17)) for the fixed point $P$ of non-conservative system (14)-(16).

$$
\tan \beta_{P}=\frac{\Gamma-2 \gamma}{\nu}, \quad a_{1 P} \sin \beta_{P}=\gamma, \quad a_{2 P}^{2} \sin ^{2} \beta_{P}=\Gamma \gamma
$$

which is stable above the line

$$
\nu^{2}=(2 \gamma-\Gamma)^{2} \frac{2 \gamma^{2}-2 \gamma \Gamma+\Gamma^{2}}{2 \gamma^{2}-2 \gamma \Gamma-\Gamma^{2}}
$$

where a Hopf bifurcation occurs. This bifurcation is supercritical to the left of the minimum in Fig. 3; the ensuing limit cycle is shown numerically to start a period-doubling cascade as $\nu$ is decreased. System (14)(16) was first analysed by Vyshkind and Rabinovich [13]; part of its rich chaotic structure was numerically detailed by Wersinger et al. [16]. An ad-hoc model for a 1D map representing the system was given by Bussac [18]. Hughes and Proctor [3] used a multiple time-scales method to derive a consistent 1D map at small, positive $\Gamma$.

\subsection{Topology of the transition}

We next study Eqs. (14)-(16) in the light of the topological discussion of Section 2. Setting $a_{2}^{2} \equiv A_{2}$ in Eqs. (14), (16), we write (15) as $\dot{A}_{2}=-2 \gamma A_{2}+2 A_{2} a_{1} \sin \beta ; A_{2}$ real may now have any sign, the invariant surface $\Sigma\left(A_{2}=0\right)$ separating half-spaces. The fixed point $P$ now exists for $\Gamma<0$ too. $P$ then lies in the unphysical half-space $\left(A_{2 P}=\Gamma \gamma / \sin ^{2} \beta_{P}<0\right)$, and the linearized flow near it has one positive and two negative eigenvalues (saddle-node with $1 \mathrm{D}$ unstable manifold). At $\Gamma=0$, the invariant sets $P$ and $\Sigma$ meet, generating the same line $\Lambda\left(A_{2}=0, \nu+2 a_{1} \cos \beta=0\right)$ of Section 2 , even though we here have $\gamma \neq 0 . P$ is now doubly-degenerate, and lies on the $\beta<\pi$ branch of $\Lambda$, at $a_{1 P}=\left(\gamma^{2}+\nu^{2} / 4\right)^{1 / 2}$ (Fig. 4). The entire orbital structure of $\Sigma$ remains, again, as in Section 2 .

The invariant surface $\Sigma^{\prime}$ of the conservative case $\left(\nu+2 a_{1} \cos \beta=0\right)$ remains, for $\Gamma=0$, too, but the orbital structure is different. Using (12) and (15), together with $\nu+2 a_{1} \cos \beta=0$, we get a new family of heteroclinic orbits, 


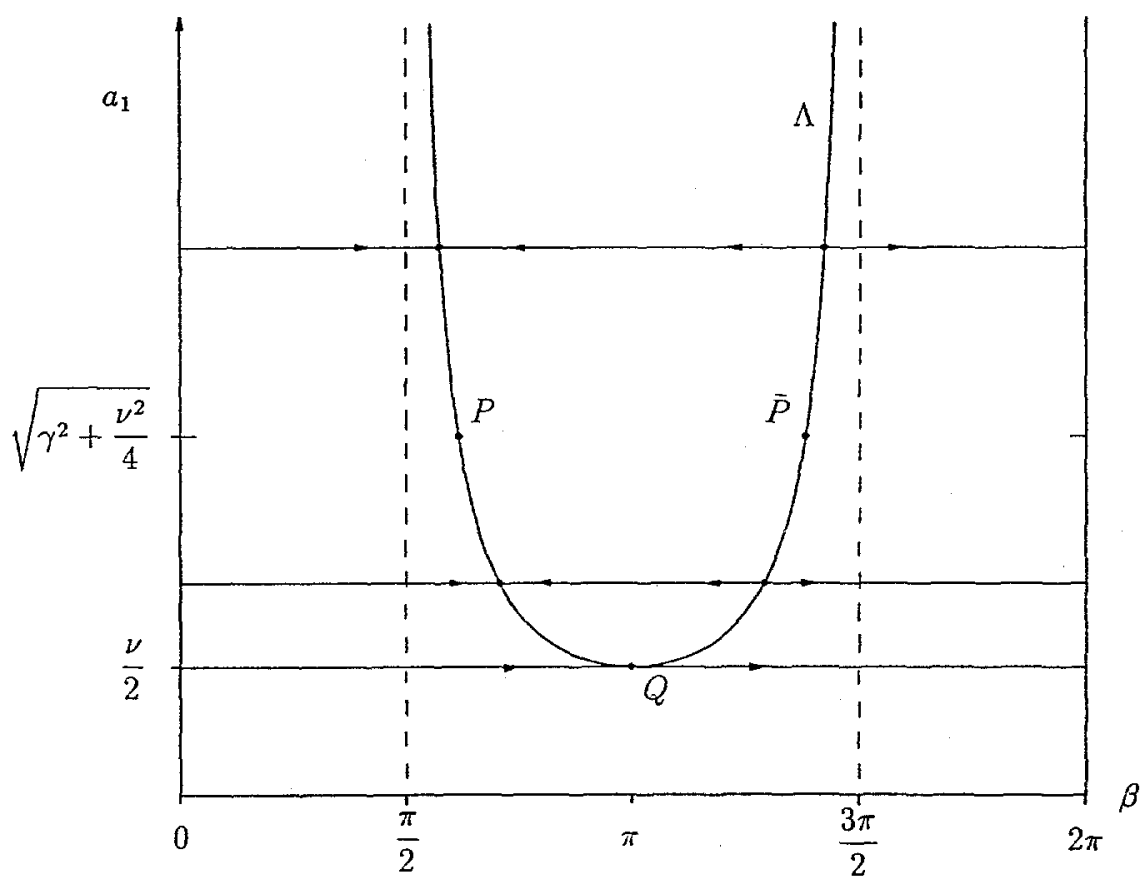

Fig. 4. Plane $a_{1}-\beta$ showing point $P$ and its symmetric $\bar{P}$ on line $A$, for zero growth rate $\Gamma$ in system (14)-(16). Fixed points in the arc $P Q$ are stable.

$$
\begin{aligned}
& a_{2}^{2}+\left(\gamma+\frac{\nu}{2} \tan \beta\right)^{2}=a_{2}^{2}+\left[\gamma \mp\left(a_{1}^{2}-\frac{\nu^{2}}{4}\right)^{1 / 2}\right]^{2}=\text { constant } \\
& \tan \beta+\frac{2 \gamma}{\nu}=\left(\tan \beta_{\infty}+\frac{2 \gamma}{\nu}\right) \tanh \left[\frac{1}{2} \nu t\left(\tan \beta_{\infty}+\frac{2 \gamma}{\nu}\right)\right]
\end{aligned}
$$

recovering Eqs. (5) and (13), respectively, as $\gamma \rightarrow 0$. The $\mp$ sign in (18) applies for $\beta \lessgtr \pi$; note that $\tan \beta_{\infty} \equiv \tan \beta(t \rightarrow \infty) \neq-\tan \beta(t \rightarrow-\infty)$. As indicated in Fig. 5, orbits starting within some range above $P$ in the $\beta<\pi$ branch end on the same branch, below $P$; points in the range $Q-P$ thus have no unstable manifold (two negative, one zero eigenvalues). Since all orbits in the $A_{2}>0$ halfspace approach the surface $\Sigma$ (as follows from $d\left(a_{1}^{2}+A_{2}\right) / d t=-2 \gamma A_{2}$ ), the attractor at $\Gamma=0$ is the range $Q-P$ of line $A$, together with the family of periodic orbits below $Q$ in Fig. 4 . A very simple relation between $a_{1 M}$ and $a_{1 m}$ for the start $(M)$ and end $(m)$ points of each heteroclinic orbit on $\Sigma^{\prime}$ follows from Eqs. (18), (19). Since $a_{2} \rightarrow 0$ as $t \rightarrow \mp \infty$, we have

$$
\gamma-\left(a_{1 M}^{2}-\nu^{2} / 4\right)^{1 / 2}=-\left[\gamma \mp\left(a_{1 m}^{2}-\nu^{2} / 4\right)^{1 / 2}\right]
$$

with sign $\mp$ for $\beta_{m}<\pi$. This may just be written as

$$
Z_{m}=\left|Z_{M}-2\right|, \quad Z \equiv\left(\frac{a_{1}^{2}}{\gamma^{2}}-\frac{\nu^{2}}{4 \gamma^{2}}\right)^{1 / 2} \geq 0
$$

$Z_{M}$ grows with $a_{1 M}$; to $Z_{M}=1(M=P), 2$ and 3 correspond $Z_{m}=1,0$ and $1(m=P, Q$ and $\bar{P}$ in Figs. 4,5), respectively. 


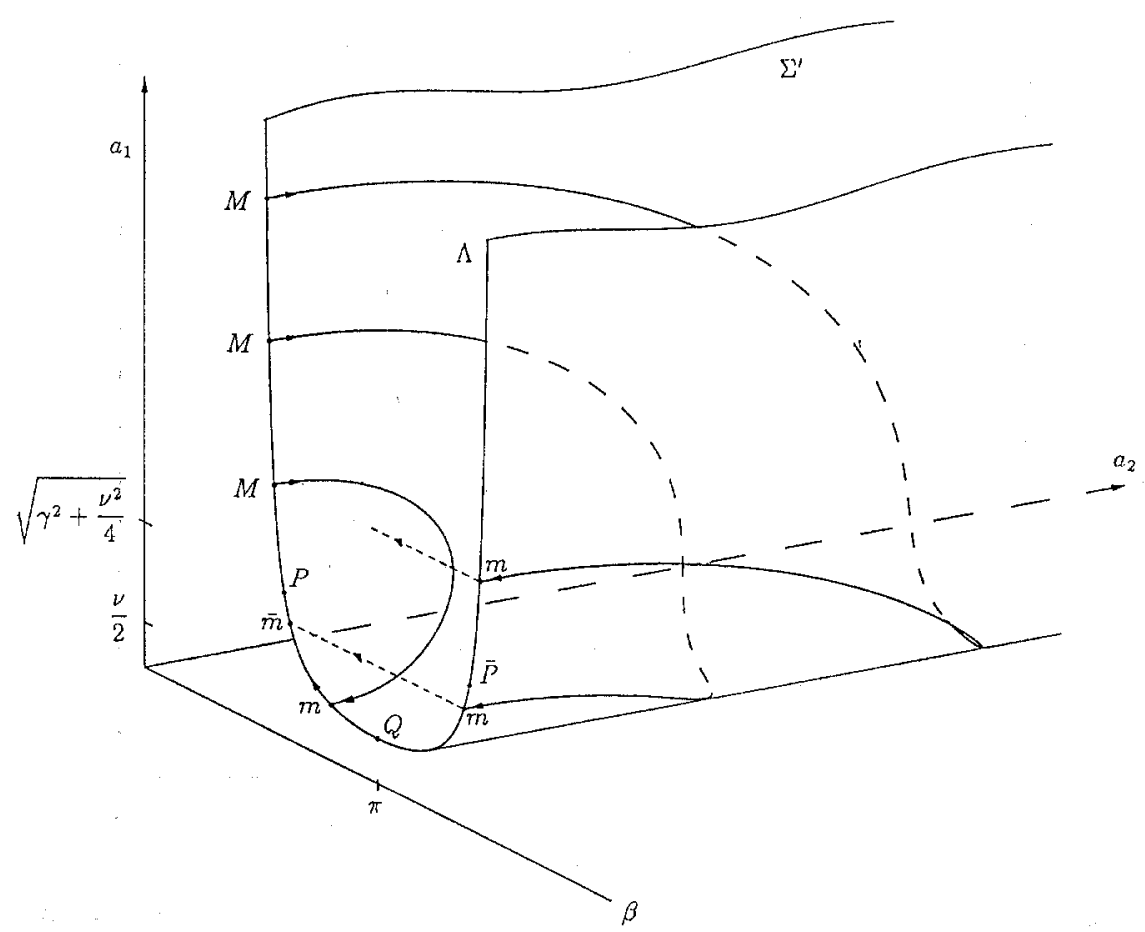

Fig. 5. Heteroclinic orbits contained in the invariant surfáce $\Sigma^{\prime}$, beginning at fixed points on $A$ above point $P$ and ending at either side of $Q$ and above or below $\bar{P}$, for zero growth rate $\Gamma$.

System (14)-(16) is, however, structurally unstable at $\Gamma=0$. Consider $\Gamma$ small and positive, and below the line in Fig. 3 given by Eq. (17); $P$ is now a saddle-focus with an unstable $2 \mathrm{D}$ manifold. For $\Gamma \rightarrow 0^{+}$, those parts of the spiraling orbits lying between $P$ and $\Sigma$ are crashed onto the line $A$. Points in the plane $a_{2}=0$ lying on $A$ between $Q$ and $P$ will drift upward on this line at a vanishing rate given by the equations

$$
\begin{aligned}
& \dot{a}_{1}=\Gamma a_{1} \\
& \dot{\beta}=\nu+2 a_{1} \cos \beta=0(\Gamma)
\end{aligned}
$$

The $\Gamma \rightarrow 0^{+}$attractor may then be described by an exact 1D map of maxima $a_{1 M}$, or $Z_{M}$.

One type of iteration in the map is made, in the limit, of two steps: a $\Sigma^{\prime}$ heteroclinic orbit, from $M$ to a point $m$ lying on $Q P$ in Fig. 5, leading to Eq. (20), and a rise on $A$, from $m$ to a new maximum $M^{\prime}$ above $P$. To describe this second step, note that, no matter how close the solution to the heteroclinic $M \rightarrow m$ orbit, Eqs. (14), (16) will ultimately read as (21), (22), at $a_{2}$ small enough. Using also Eq. (15), $\dot{a}_{2} / a_{2}=-\gamma+a_{1} \sin \beta$, one obtains

$$
\begin{aligned}
& \ln \frac{a_{2 r}}{a_{2}}=\frac{\gamma}{\Gamma}\left[F\left(Z_{r}, \frac{\nu}{\gamma}\right)-F\left(Z, \frac{\nu}{\gamma}\right)\right] \\
& F\left(Z, \frac{\nu}{\gamma}\right) \equiv Z-\frac{1}{2} \ln \left(1+\frac{4 \gamma^{2}}{\nu^{2}} Z^{2}\right)-\frac{\nu}{2 \gamma} \tan ^{-1}\left(\frac{2 \gamma}{\nu} Z\right)
\end{aligned}
$$

with $a_{2 r}$ a small reference value. Moving up on $\Lambda, a_{2}$ will first decrease ( $a_{1 r} \simeq a_{1 m}$ is less than $a_{1 P}, \dot{a}_{2}$ changing sign at $a_{1}=a_{1 p}$ ). At vanishing $\Gamma$, the ratio $a_{2} / a_{2 r}$ will then be exponentially small, $\dot{a}_{1}$ remaining positive until a value $a_{1 M^{\prime}}$ satisfying 


$$
F\left(Z_{M^{\prime}}, \nu / \gamma\right)=F\left(Z_{m}, \nu / \gamma\right)
$$

is reached; any effect from a difference $a_{1 r}-a_{1 m}$ vanishes with $\Gamma$. Using Eqs. (20) and (23), and writing $Z_{M} \equiv Z_{M, n}, Z_{M^{\prime}} \equiv Z_{M, n+1}$, the overall map then reads

$$
F\left(Z_{M, n+1}, \nu / \gamma\right)=F\left(\left|Z_{M, n}-2\right|, \nu / \gamma\right)
$$

For $M$ such that $m$ lies on the $\beta>\pi A$-branch between $Q$ and $\bar{P}\left(2<Z_{M}<3\right.$, Fig. 5), the iteration involves three steps; a middle step, before the rise on $A$, moves along a heteroclinic orbit on $\Sigma$, from $m$ to its symmetric point $\bar{m}$ on the $\beta<\pi$ branch (the $a_{1}$ variation vanishing with $\Gamma$ ). Eqs. (23) and (24) still hold. Finally, for $Z_{M}>3$ ( $m$ above $\bar{P}$ ), the iteration again involves two steps. Since we have $a_{1 \bar{m}}=a_{1 m}>a_{1 P}, a_{2}$ is already increasing at point $\bar{m}$, and the new iteration starts just at $\bar{m}$; we then have

$$
\begin{aligned}
& Z_{M^{\prime}}=Z_{m} \\
& Z_{M, n+1}=\left|Z_{M, n}-2\right|
\end{aligned}
$$

Eqs. (23),(24) and (25), (26) thus apply for $Z_{M} \leq 3$, and $Z_{M} \geq 3$, respectively.

Fig. 6 shows the full map, (24),(26), at $\nu=0.655 \gamma$ [3]. The slope near point $(1,1)$,

$$
\frac{d Z_{M, n-1}}{d Z_{M, n}} \simeq 1+\frac{4}{3} \frac{4 \gamma^{2}-\nu^{2}}{4 \gamma^{2}+\nu^{2}}\left(Z_{M, n}-1\right)
$$

recovers the loss of stability for the fixed point $P$ of the flow (14)-(16) at $\nu=2 \gamma$ (Fig. 3). One may show analytically that the fixed point $N$ of the map in Fig. 6 (the first limit cycle of the flow for $\Gamma \rightarrow 0^{+}$) loses stability at $\nu \simeq 0.932 \gamma$, starting a period-doubling cascade that ends in chaos. The return time for the map, $t_{M M^{\prime}}$, is dominated by the rise on $\Lambda$ for $Z_{M}<3$; using (21) we find

$$
t_{M M^{\prime}}\left(\Gamma, Z_{M}, \frac{\nu}{\gamma}\right) \simeq \frac{1}{2 \Gamma} \ln \frac{Z_{M^{\prime}}^{2}+\nu^{2} / 4 \gamma^{2}}{Z_{m}^{2}+\nu^{2} / 4 \gamma^{2}}=0\left(\frac{1}{\Gamma}\right)
$$

For $Z_{M}>3$, with no $\Lambda$ rise, one finds, from the heteroclinic orbits $M \rightarrow m \rightarrow \bar{m}, t_{M M^{\prime}}=0[\ln (1 / \Gamma)][19]$. For further discussion see Section 5.

\section{Generic conditions for the hard transition}

We first show that coupled oscillators with frequencies near the ratio $2: q, q$ any integer, exhibit the transition of Section 3 for $q=1$. Consider the general resonance $n: q$ and set

$$
x \propto a_{1} \cos \left(n \omega_{0} t+\psi\right), \quad y \propto a_{2} \cos \left(q \omega_{0} t+\theta\right)
$$

The equations for $a_{1}, a_{2}$ will always take the form

$$
\dot{a}_{1}=\Gamma a_{1}-a_{2} F, \quad \dot{a}_{2}=-\gamma a_{2}+a_{1} F
$$

for some appropriate function $F$ because, for $\Gamma=\gamma=0$, the (conservative) coupling should preserve the action $a_{1}^{2}+a_{2}^{2} ; F$ must clearly be a periodic function of $\beta \equiv n \theta-q \psi$, with a polynomic dependence on both $a_{1}$ and $a_{2}$. If (i) $F$ vanishes with $a_{2}$ and (ii) $f \equiv F / a_{2}$ does not, the resulting system 


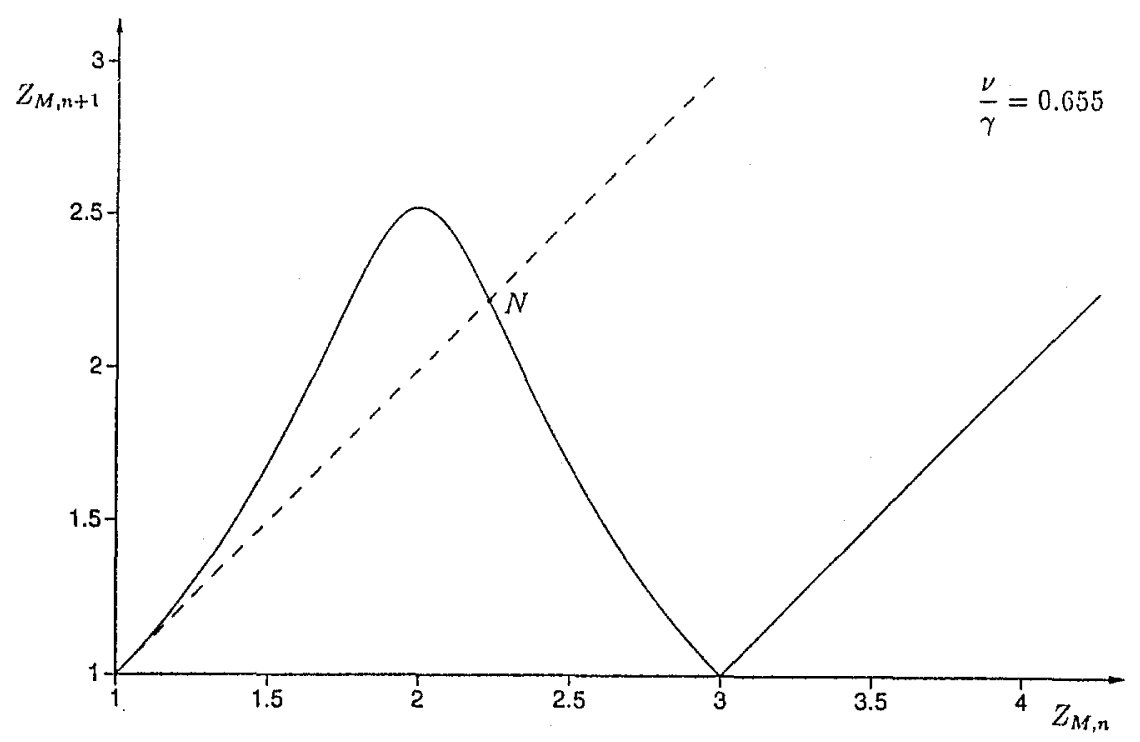

Fig. 6. Map of maxima $Z_{M}$ as obtained from Eqs. (24),(26), with $Z$ defined in (20), for non-conservative system (14)-(16) and vanishing growth rate, $\Gamma \rightarrow 0^{+}$.

$$
\begin{aligned}
& \dot{a}_{1}=\Gamma a_{1}-a_{2}^{2} f \\
& \dot{a}_{2}=-\gamma a_{2}+a_{1} a_{2} f \\
& \dot{\beta}=h\left(a_{1}, a_{2}, \beta\right)
\end{aligned}
$$

with $h$ again periodic in $\beta$, has, generically, a number of properties in common with system (14) $-(16)$ for the $2: 1$ resonance:

(1) The surface $a_{2}=0 \quad(\Sigma)$ is an invariant surface.

(2) For $\Gamma=0$, there is a line $(\Lambda)$ of fixed points on $\Sigma$ : $a_{2}=0, h\left(a_{1}, 0, \beta\right)=0$.

(3) For $\Gamma$ positive and small enough, there is a fixed point $P$ (different from the rest state, $a_{1}=a_{2}=0$ ) that lands on $A$ as $\Gamma \rightarrow 0^{+}$,

$$
\begin{aligned}
& a_{2}^{2}=\gamma \Gamma / f^{2} \rightarrow 0^{+} \\
& a_{1} f\left(a_{1}, 0, \beta\right)=\gamma \\
& h\left(a_{1}, 0, \beta\right)=0
\end{aligned}
$$

(4) Preservation of phase volume by the nonlinear terms takes here the form

$$
\frac{\partial h}{\partial \beta}=-2 a_{1} f-a_{2} a_{1} \frac{\partial f}{\partial a_{2}}+\frac{a_{2}^{2}}{a_{1}} \frac{\partial}{\partial a_{1}}\left(a_{1} f\right)
$$

and yields $\partial h /\left.\partial \beta\right|_{a_{2}=0}=-2 a_{1} f\left(a_{1}, 0, \beta\right)$. Clearly, neither $\partial h / \partial \beta$, nor $\partial h / \partial a_{1}, \partial^{2} h / \partial \beta^{2}$ or $d a_{1} /\left.d \beta\right|_{\Lambda}$ vanishes identically with $a_{2}$. For $\Gamma=0$, the flow on $\Sigma$, given by $\dot{a}_{1}=0 \rightarrow a_{1}=a_{10}$ and $\dot{\beta}=h\left(a_{10}, 0, \beta\right)$, will then exhibit a saddle-node bifurcation at a point $Q$ on $\Lambda$ given by

$$
\begin{aligned}
& h\left(a_{1 Q}, 0, \beta_{Q}\right)=0 \\
& \frac{\partial h}{\partial \beta}\left(a_{1 Q}, 0, \beta_{Q}\right)=0
\end{aligned}
$$


As in Fig. 4, $Q$ will be a homoclinic point with a (double) familiy of heteroclinic orbits joining the two branches of $\Lambda$ (and a family of periodic orbits before bifurcation).

To satisfy conditions (i), (ii), a term $x^{s} y^{2}$ in the expansion of the potential $V(x, y)$ must couple to the unstable (driven) $x$-oscillator. The strongest resonance occurs for $s+1=2 q / n$; a term $x^{s+1} y$ now couples to the $y$-oscillator. One may then set $n=2$, leading to $q(=s+1)=1,2,3, \ldots$ Writing $V_{x x}^{0} \equiv 4 m \omega_{0}^{2}, V_{y y}^{0} \equiv$ $m\left(q \omega_{0}+\nu / 2\right)^{2}, \nu / \omega_{0}$ small, proceeding as in Section 2 and scaling $x$ and $y$ conveniently, we obtain, for $q \neq 2$,

$$
\begin{aligned}
& f=a_{1}^{q-1} \sin \beta \\
& h=\nu+a_{1}^{q-1}\left(2 a_{1}-q \frac{a_{2}^{2}}{a_{1}}\right) \cos \beta+C a_{1}^{2}+D a_{2}^{2}
\end{aligned}
$$

$f$ indeed satisfies (i) and (ii). The term $C a_{1}^{2}\left(D a_{2}^{2}\right)$ in $h$ arises from cubic terms $x^{3}$ and $x y^{2}\left(x^{2} y\right.$ and $\left.y^{3}\right)$ in the expansion for $V$, which always couple to the $x(y)$ oscillator; to recover system (14)-(16) for $q=1$ one drops those terms which are then of higher order. Although the opposite holds for $q>2$, the full expression for $h$ must now be used because parameters $C$ and $D$ enter only the rate $\dot{\beta}$ and may be of arbitrary value.

The $q=2$ case (equivalent to resonance $1: 1$ ) is peculiar because cubic terms then produce the entire expressions for $h$ and $f$. For $q=2$, we find

$$
\begin{aligned}
& f=a_{1} \sin \beta-\left(\bar{V}_{1} \frac{a_{1}^{2}}{a_{2}}+\bar{V}_{3} a_{2}\right) \sin \frac{1}{2} \beta \\
& h=\nu+a_{1}\left(2 a_{1}-2 \frac{a_{2}^{2}}{a_{1}}\right) \cos \beta+C a_{1}^{2}+D a_{2}^{2}+6\left(\bar{V}_{3}-\bar{V}_{1}\right) a_{1} a_{2} \cos \beta+2\left(\bar{V}_{1} \frac{a_{1}^{3}}{a_{2}}-\bar{V}_{3} \frac{a_{2}^{3}}{a_{1}}\right) \cos \frac{1}{2} \beta
\end{aligned}
$$

$C$ and $D$ here taking the values

$$
\begin{aligned}
& C=2\left(2-\bar{V}_{0}\right) \\
& D=2\left(\bar{V}_{4}-2\right)
\end{aligned}
$$

We wrote $\bar{V}_{j}=V_{j} / V_{2} ; V_{x x x x}^{0} \equiv V_{0}, V_{x x x y}^{0} \equiv V_{1}$, etc.; and

$$
\begin{aligned}
& x \equiv\left(32 m \omega_{0} / V_{2}\right)^{1 / 2} a_{1} \cos \left(2 \omega_{0} t+\psi\right) \\
& y \equiv\left(32 m \omega_{0} / V_{2}\right)^{1 / 2} a_{2} \cos \left(2 \omega_{0} t+\theta\right)
\end{aligned}
$$

For $f$ here to satisfy condition (i), $\bar{V}_{1}$ must clearly be zero; we shall further take $\bar{V}_{3}=0$ for simplicity (see Section 5). We may then use expressions (36), (37) for all $q$, just setting $C=D=0$ for $q=1$.

Eqs. (27)-(29) with $f$ and $h$ given by (36),(37) present a new property, (5), and stronger forms of properties (3) and (4):

(5) For $f$ independent of $a_{2}$ and $h$ linear in $a_{2}^{2} \quad\left[h=h_{0}\left(a_{1}, \beta\right)+a_{2}^{2} h_{2}\left(a_{1}, \beta\right)\right]$ as in (36), (37), Eq. (33), representing nonlinear preservation of phase volume, is equivalent to

$$
\frac{\partial h_{0}}{\partial \beta}=-2 a_{1} f, \quad \frac{\partial}{\partial \beta}\left(h_{2}+\frac{1}{2 a_{1}} \frac{\partial h_{0}}{\partial a_{1}}\right)=0
$$

If, actually, the last condition takes a stronger form,

$$
h_{2}+\frac{1}{2 a_{1}} \frac{\partial h_{0}}{\partial a_{1}}=0
$$


a second invariant surface $\Sigma^{\prime}\left[h_{0}\left(a_{1}, \beta\right)=0\right]$ exists at $\Gamma=0$ : on the surface $\Sigma^{\prime}$ we do then have

$$
\frac{\partial h_{0}}{\partial \beta} \dot{\beta}+\frac{\partial h_{0}}{\partial a_{1}} \dot{a}_{1}=a_{2}^{2}\left(h_{2} \frac{\partial h_{0}}{\partial \beta}-f \frac{\partial h_{0}}{\partial a_{1}}\right) \equiv 0
$$

For expressions (36),(37), condition (42) reads $C+D=0$, certainly satisfied for $q=1$.

(3') Using (36),(37) in Eqs. (31),(32) for the fixed point $P$ at vanishing $\Gamma$, one finds, for $q>1$, $\beta_{P}\left(\nu / \gamma, C \gamma^{(2-q) / q}\right)$ and $a_{1 P}^{q}=\gamma / \sin \beta_{P}$ (there may be more than one solution, or no solution, to (31),(32); see Section 5). We assume $\nu>0$; otherwise write $\nu, \beta, C, D \rightarrow-\nu, \pi-\beta,-C,-D$. The eigenvalues for the linearized flow at $P$ are $\lambda=-2 \gamma$ and

$$
\begin{aligned}
\lambda \simeq & \pm i(2 \gamma \Gamma)^{1 / 2}\left[\frac{q-2 \cos \beta_{P}\left[(\nu / 2 \gamma) \sin \beta_{P}+\cos \beta_{P}\right]}{\sin ^{2} \beta_{P}}\right]^{1 / 2} \\
& +\frac{\Gamma}{2}\left[2-\frac{q+(D / C) 2 \cos \beta_{P}\left[(\nu / 2 \gamma) \sin \beta_{P}+\cos \beta_{P}\right]}{\sin ^{2} \beta_{P}}\right]
\end{aligned}
$$

Clearly, $P$ will be a saddle-focus with a $2 D$ unstable manifold, as in Section 3, within certain parameter domain that includes a subdomain for $D+C=0$, reading

$$
q-2<\frac{\nu}{2 \gamma} \sin 2 \beta_{P}<q-2 \cos ^{2} \beta_{P}
$$

For $\beta_{P}$ and $\nu / \gamma$ satisfying these two conditions, Eqs. (31),(32) and (36),(37) determine $C \gamma^{(2-q) / q}$ and $a_{1 P}^{2} / \gamma$; one finds $\beta_{P} \leq \pi / 2, C<0$, and $\nu>2 \gamma(q-2)$. Note that $P$ is doubly degenerate at $\Gamma=0$, the eigenvalue $\lambda=-2$ showing that fixed points on the $\beta<\pi$ branch of $\Lambda$ are stable on $\Sigma$, as in Fig. 4; for $\Gamma<0$ ( $A_{2 P} \equiv a_{2 P}^{2}$ negative), $P$ is a saddle-node with a $1 D$ unstable manifold, as in Section 3 again.

(4') Using (37) in Eqs. (34),(35) we find, for $q>1,2 a_{1 Q}^{q}-C a_{1 Q}^{2}=\nu$ and $\beta_{Q}=\pi$ (for a bifurcation at $\beta=0$ or $2 \pi$, see Section 5). Since $\nu$ is positive, $a_{1 Q}$ does exist for $C<0$. Near $Q$ one then has

$$
\left.\frac{d a_{1}}{d \beta}\right|_{\Lambda} \simeq \frac{a_{1 Q}^{q+1}\left(\beta-\beta_{Q}\right)}{\nu+(q-2) a_{1 Q}^{q}}
$$

proving $d^{2} a_{1} /\left.d \beta^{2}\right|_{\Lambda}$ positive at $Q$, as in Fig. 4 , for all $q$. This means that the upward drift on $A$ at vanishing rate when $\Gamma \rightarrow 0^{+}$, as given by Eq. (21), takes the solution off the stable range $P Q$ of the $\beta<\pi$ branch of $\Lambda$, again as in the $q=1$ case.

From properties $(1),(2),\left(3^{\prime}\right),\left(4^{\prime}\right)$ and (5) we conclude that, if $D=-C$, and for appropriate ranges of the dimensionless parameters $\nu / \gamma, C \gamma^{(2-q) / q}$, all resonances $2: q$, described by Eqs. (27)-(29) and (36),(37), will exhibit at $\Gamma=0$ the topology and hard transition to chaos found in case $q=1$. Two examples are discussed in next section. With fixed point $P$ a saddle-focus, the transition will exist even if there is no surface $\Sigma^{\prime}$ $\left(D \neq-C\right.$ ) because, as $\Gamma \rightarrow 0^{+}$and for times long enough, the equation

$$
\frac{d}{d t}\left(a_{1}^{2}+a_{2}^{2}\right)=-2 \gamma a_{2}^{2}
$$

will bring $a_{2}^{2}$ below $\Gamma$, no matter how small, making operative the heteroclinic structure tied to line $A$ and point $P$. (The absence of $\Sigma^{\prime}$ just makes impossible a quick determination of the exact 1D map for the attractor, as carried out in Sections 3 and $5 ; \Sigma^{\prime}$ is absent, for instance, in the nonlinear Schrödinger equation under 3-wave truncation [20], which has $\bar{V}_{1}=\bar{V}_{3}=0, \bar{V}_{0}=3, \bar{V}_{4}=7 / 2$ in Eqs. (38),(39).) 
Actually, any system (27)-(29) with $f$ meeting conditions (i) and (ii) and thus showing properties (1)(4), whether arising from a resonance (say the $2: 2$ case with $V_{3} \neq 0$ ) or not, should exhibit the transition on some parametric domain where the stronger properties $\left(3^{\prime}\right),\left(4^{\prime}\right)$ would hold. In particular, we expect to find the transition in wave interactions with $\underline{3}$ different amplitudes, and in rings of oscillators, a model of interest in biophysics [21]. We note, finally, that resonances $n: 1$ will exhibit the transition for $n=1,2$, (identical to resonances $2: q, q=2,1$ ), but not for $n>2$, when $f$ does not satisfy condition (ii); the $n=3$ case represents the interaction of a wave and its third harmonic [10].

\section{Highly symmetric $1: 1$ resonance}

Here we consider the rich $2: q, q=2$ resonance for a potential energy $V(x, y)$ even and symmetrical in $x$ and $y$. We do then have $\bar{V}_{1}=\bar{V}_{3}=0$ and $\tilde{V}_{0}=\bar{V}_{4}(D=-C)$ in Eqs. (38),(39), and arrive at system

$$
\begin{aligned}
& \dot{a}_{1}=\Gamma a_{1}-a_{2}^{2} a_{1} \sin \beta \\
& \dot{a}_{2}=-\gamma a_{2}+a_{2} a_{1}^{2} \sin \beta \\
& \dot{\beta}=\nu-2\left(a_{1}^{2}-a_{2}^{2}\right)(\bar{V}-\cos \beta), \quad \bar{V} \equiv \bar{V}_{0}-2=-C / 2
\end{aligned}
$$

The mismatch $\nu$ could arise from a small departure from symmetry retained in the frequencies but neglected in the weak nonlinear terms, which involve the fourth order $V$-derivatives. Alternatively, the defective feedback model suggested in Section 3 would need no frequency mismatch, the $\nu$-term arising from a phase mismatch in the excitation.

The surface $\Sigma^{\prime}$ at $\Gamma=0$ would be

$$
h_{0}\left(a_{1}, \beta\right) \equiv \nu-2 a_{1}^{2}(\vec{V}-\cos \beta)=0
$$

For $\bar{V}<-1$ neither $\Sigma^{\prime}$ nor line $\Lambda$ exist, because $h_{0}\left(a_{1}, \beta\right)$ will vanish nowhere; there is, therefore, no hard transition to chaos. For $-1<\bar{V} \leq 1, \Lambda$ behaves as in Fig. 4, the vertical asymptotes moving from the vicinity of $\pi$, for $\bar{V}$ close to -1 , to 0 and $2 \pi$, for $\bar{V}=1$. Eqs. (30)-(32) for the fixed point $P$ at vanishing $\Gamma$ give

$$
a_{1 P}^{2}=\frac{-\bar{V} \nu \pm\left[\nu^{2}+4 \gamma^{2}\left(1-\bar{V}^{2}\right)\right]^{1 / 2}}{2\left(1-\bar{V}^{2}\right)}
$$

with the upper sign. The map for the chaotic attractor resembles the $2: 1$ map of Fig. 6 .

Whenever $V(x, y)$ is a function of just $x^{2}+y^{2}$ one has $\bar{V}=1$. Eqs. (44),(45) and (46) then lead to equations for $(\beta+\pi) / 2, \nu^{1 / 2} a_{1}$ and $\nu^{1 / 2} a_{2}$ identical to those describing the $\Sigma^{\prime}$ heteroclinic orbits of Section 3; hence, we recover Eq. (20) with $\nu a_{1}^{2}$ replacing $a_{1}^{2}$,

$$
Z_{m}=\left|Z_{M}-2\right|, \quad Z \equiv\left(\frac{\nu a_{1}^{2}}{\gamma^{2}}-\frac{\nu^{2}}{4 \gamma^{2}}\right)^{1 / 2}
$$

We similarly recover Eqs. (23),(25) for the other steps in the attractor, and thus the same complete $\Gamma \rightarrow 0^{+}$map of the $2: 1$ resonance, Eqs. (24),(26). A rigorous and detailed derivation of the map was given by Sanmartín et al. [19], who studied system (43)-(45) with $\bar{V}=1$ for the weak oscillations of a spherical pendulum, under the defective feedback model. They gave exact results on superstable and degenerate homoclinic orbits in the map, and found analytically the corrections of order $\Gamma / \gamma$ and $(\Gamma / \gamma) \ln (\gamma / \Gamma)$, for $\nu / \gamma \rightarrow 0^{+}$. Note that systems (43)-(45), $\bar{V}=1$, and (14)-(16), having identical $\Gamma \rightarrow 0^{+}$maps, remain different in the limit: $h_{0}$ 


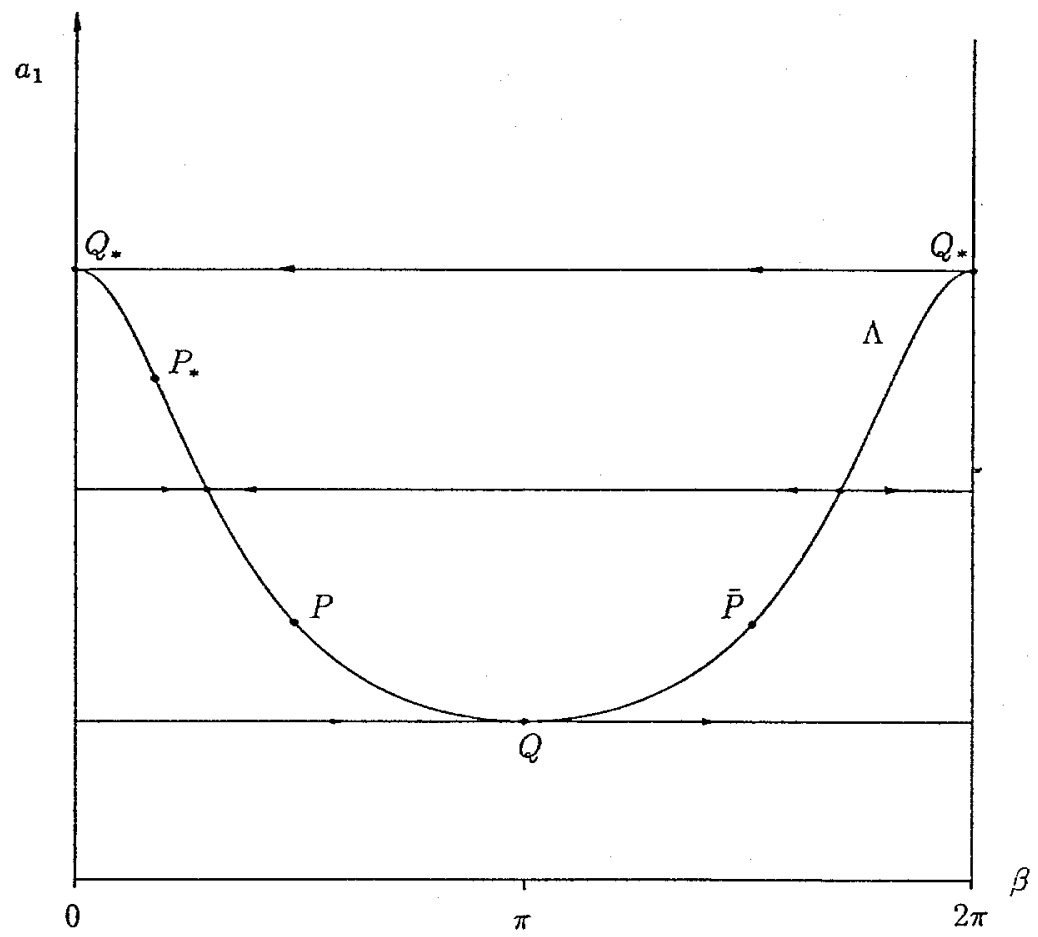

Fig. 7. Plane $a_{1}-\beta$ showing the line $A$ of fixed points for $1: 1$ resonance, with parameter $\bar{V}$ above 1 and zero growth rate $\Gamma$ in Eqs. (43)-(45). Points in the segments $P Q$ and $P_{*} Q_{*}$ are stable.

in (46) will only vanish for $\nu>0$ (in the defective feedback model, chaos may set in only if the excitation leads the response [19]), whereas $h_{0} \equiv \nu+2 a_{1} \cos \beta$ in (16) will vanish for both $\nu>0$ and $\nu<0$.

For $\bar{V}>1$ Eq. (47) yields two positive values for $a_{1}^{2}$, the second fixed point, $P_{*}$, being a saddle-node with a 1D unstable manifold. There is also a second bifurcation point $Q_{*}$ at $\beta=0(2 \pi)$, with $a_{1}$ finite throughout. Fig. 7 shows $A$ schematically for $\bar{V}>1$. Since there are no fixed points for $\bar{V}^{2}>1+\nu^{2} / 4 \gamma^{2}$, we consider values

$$
0<\delta^{2} \equiv 4 \gamma^{2}\left(\bar{V}^{2}-1\right) / \nu^{2}<1
$$

Comparing Figs. 4 and 7 , for $\Gamma=0$, we note that two ranges of $\Lambda, P Q$ and $P_{*} Q_{*}$, now have no unstable manifold (also, there are periodic orbits below and above $\Lambda$ ). Heteroclinic orbits leaving the branch $\beta<\pi$ must thus start in the range $P P_{*}$; hence, the map of maxima $a_{1 M}$ describing the $\Gamma \rightarrow 0^{+}$attractor will be defined in the interval $\left[a_{1 P}, a_{1 P_{*}}\right]$ only.

As in Section 3, each map iteration has a first step relating values $a_{1 M}$ and $a_{1 m}$ for the start $(M)$ and end ( $m$ ) points of a $\Sigma^{\prime}$ heteroclinic orbit moving from range $P P_{*}$ to some $\Lambda$ point to the right of $P$. Proceeding as for resonances $2: 1$, and $1: 1$ with $\bar{V}=1$, we find

$$
\frac{\zeta_{M}-\zeta_{m}}{\sin ^{-1} \zeta_{M}-\sin ^{-1} \zeta_{m}}=\delta, \quad \zeta \equiv \frac{2}{\nu} a_{1}^{2}\left(\bar{V}^{2}-1\right)-\bar{V}
$$

yielding $\zeta_{m}\left(\zeta_{M}, \delta\right)$. (For $\bar{V}-1 \rightarrow 0^{+}$at fixed $\nu / \gamma, \sin ^{-1} \zeta_{m}+\pi / 2$ vanishes with either sign, and Eq. (48) follows). The $\zeta$ variable takes values $-1,-\left(1-\delta^{2}\right)^{1 / 2}$ and $+\left(1-\delta^{2}\right)^{1 / 2}$ at points $Q, P$ and $P_{*}$, respectively. Eq. (49) then yields $m=P$ at $M=P$, and makes $\zeta_{m}$ first decreasing when increasing values of $\zeta_{M}$ are 
considered; $m$ reaches $Q$ with $M$ at $P_{*}$, for $\delta \simeq 0.725$. Hence, there may be no hard transition within the range $0.725<\delta<1, m$ then lying in the arc $P Q$ for any $a_{1 M}$ in the interval $\left[a_{1 P}, a_{1 P_{*}}\right]$.

For the second part of the iteration, from $\zeta_{m}$ to a new maximum $\zeta_{M^{\prime}}$, there are three different cases, as in Section 3. For $m$ between $Q$ and the point $\bar{P}$ symmetric of $P$, the solution first moves from $m$ to $\bar{m}$ along a $\Sigma$ heteroclinic orbit, then rises on $\Lambda$ up to $M^{\prime}$, with $a_{1}$ given by Eq. (21), and $a_{2}$ decreasing from $\bar{m}$ to $P$ and increasing from $P$ to $M^{\prime}$, along the rise; point $M^{\prime}$ is given by

$$
\begin{aligned}
& G\left(\zeta_{M^{\prime}}, \delta, \bar{V}\right)=G\left(\zeta_{m}, \delta, \bar{V}\right) \\
& G \equiv \bar{V} \sin ^{-1} \zeta-\delta \ln (\zeta+\bar{V})+\left(1-\zeta^{2}\right)^{1 / 2}-\left(\bar{V}^{2}-1\right)^{1 / 2} \sin ^{-1}\left(\frac{\bar{V} \zeta+1}{\zeta+\bar{V}}\right)
\end{aligned}
$$

For $m$ between $P$ and $Q$ there is no $m \rightarrow \bar{m}$ motion but (51) holds. For $m$ above $\bar{P}$, there is no rise on $A$; instead of (51) we then have

$$
\zeta_{M^{\prime}}=\zeta_{m}
$$

Eqs. (49) and (51),(52) determine a map $\zeta_{M, n+1}\left(\zeta_{M, n}, \delta, \bar{V}\right)$ which depends on two dimensionless parameters but is similar, otherwise, to that shown in Fig. 6.

The fact that the map is defined in a bounded interval has an important consequence, however. For $m=Q$ and $\bar{V}$ fixed, Eq. (51) makes $\zeta_{M^{\prime}}$ increase as $\delta$ (or $\gamma / \nu$ ) is increased; $M^{\prime}$ then reaches $P_{*}$ at a value $\delta_{*}<\overline{0.725}$ if $\bar{V}$ is less than $1.23, \delta_{*}(\bar{V})$ being given by the equation

$$
G\left[\left(1-\delta_{*}^{2}\right)^{1 / 2}, \delta_{*}, \bar{V}\right]=G\left[-1, \delta_{*}, \bar{V}\right]
$$

At such values of $\bar{V}$, and $\delta$ in the range $\delta_{*}(\bar{V})<\delta<0.725$, one has

$$
G\left[\zeta_{P_{*}}, \delta, \bar{V}\right]<G\left[\zeta_{Q}, \delta, \bar{V}\right]
$$

Then, for $m$ within some $A$-arc enclosing $Q, a_{2}$ is still exponentially small when the solution arrives at $P_{*}, a_{2}$ decreasing and $\dot{a}_{1}$ remaining positive thereafter. As a result, the map will not be defined within some subinterval of the interval $\left[a_{1 P}, a_{1 P_{*}}\right]$, Fig. 8. The attractor is now a repellor; after a sufficient number of map iterations, all points in the interval $\left[a_{1 P}, a_{1 P_{*}}\right]$, except a Cantor set, leave the interval [22]. This is an example of crisis [23]: the attractor disappears when it meets the unstable fixed point $P_{*}$.

\section{Summary of results}

We have presented and studied a hard transition to chaos in open, dissipative systems described by flows. For positive values of a parameter $\Gamma$, no matter how small, a fully developed chaotic attractor exists within some domain of additional parameters, whereas no chaotic behavior exists for $\Gamma \leq 0$. As $\Gamma$ is made positive, a saddle-node fixed point reaches an invariant plane to enter as saddle-focus a phase half-space of physical solutions; the ghosts of a line of fixed points and a rich heteroclinic structure existing at $\Gamma=0$ make the limits $t \rightarrow+\infty, \Gamma \rightarrow+0$ non-commuting, and allow an exact description of the chaotic flow. We have fully determined the formal structure of the class of flows exhibiting the transition.

A subclass of such flows (coupled oscillators in near-resonance at any $2: q$ frequency ratio, with $\Gamma$ representing linear excitation of the first oscillator) has been analysed. We gave a detailed discussion of cases $q=1$ and $q=2$ (the rich $1: 1$ resonance), showing the variety of chaotic attractors found at transition. This transition appears to have broad applicability, in particular to wave interactions (with three or more different amplitudes) and to rings of oscillators as biological models. 


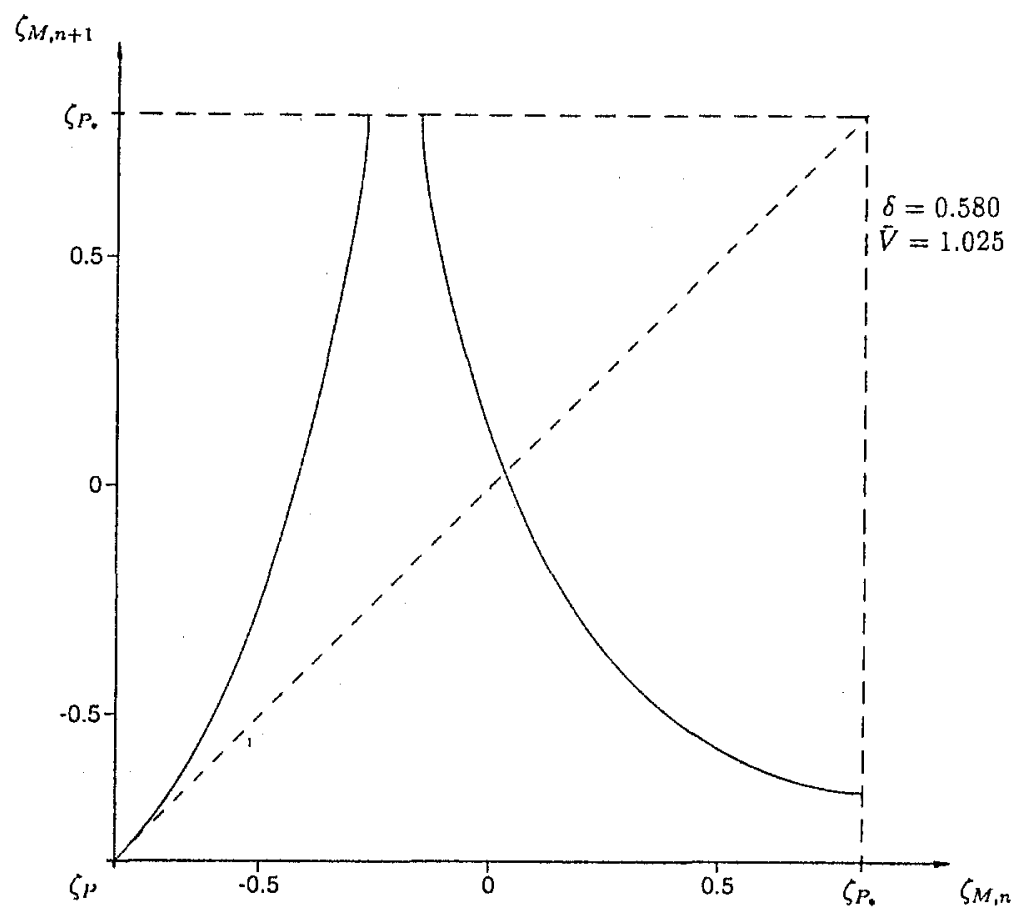

Fig. 8. Map of maxima $\zeta_{M}$ as obtained from Eqs. (49)-(51), $\zeta$ defined in (49), for the case of Fig. 7 and particular values of $\bar{V}$ and $\delta$ (or $\nu / \gamma$ ), and vanishing growth rate, $\Gamma \rightarrow 0^{+}$.

\section{Acknowledgements}

This work was supported by the Comisión Interministerial de Ciencia y Tecnología of Spain (ESP92-0989. E). Parts of this work were carried out in partial fulfilment of the requirements for the Ph.D. degree of one author (OLR).

\section{References}

[1] A. Arneodo, P.H. Coullet, E.A. Spiegel and C. Tresser, Physica D 14 (1985) 327.

[2] A.C. Fowler and M.J. McGuinness, Physica D 5 (1982) 149.

[3] D.W. Hughes and M.R.E. Proctor, Physica D 46 (1990) 163.

[4] R.C. Davidson, Methods in Nonlinear Plasma Theory (Academic Press, New York, 1972) Section 6.4.4.

[5] J. Weiland and H. Wilhelmsson, Coherent Nonlinear Interaction of Waves in Plasmas (Pergamon, Oxford, 1977) Chs. 7, 9, 10 and 12.

[6] D.J. Kaup, A. Reiman and A. Bers, Rev. Mod. Phys. 51 (1979) 275.

[7] C.C. Chow, A. Bers and A.K. Ram, Plasma Phys. Control. Fusion 34 (1992) 1945.

[8] J. Guckenheimer and A. Mahalov, Physica D 54 (1992) 267.

[9] A. Jurkus and P.N. Robson, Proc. IEEE 107b (1960) 119.

[10] J.A. Armstrong, N. Bloembergen, J. Ducuing and P.S. Pershan, Phys. Rev. 127 (1962) 1918.

[11] E. Breitenberger and R.D. Mueller, J. Math. Phys. 22 (1981) 1196;

R. Cuerno, A.F. Rañada and J.J. Ruiz-Lorenzo, Am. J. Phys. 60 (1992) 73.

[12] T.H. Stix, Waves in Plasmas (Amer. Inst. Phys., New York, 1992) Ch. 4.

[13] S.Ya. Vyshkind and M.I. Rabinovich, Sov. Phys. JETP 44 (1976) 292.

[14] A.S. Pikovsky and M.I. Rabinovich, Physica D 2 (1981) 8.

[15] J.M. Wersinger, J.M. Finn and E. Ott, Phys. Rev. Lett. 44 (1980) 453. 
[ 16$]$ J.M. Wersinger, J.M. Finn and E. Ott, Phys. Fluids 23 (1980) 1142.

[17] C. Meunier, M.N. Bussac and G. Laval, Physica D 4 (1982) 236.

[ 18] M.N. Bussac, Phys. Rev. Lett. 49 (1982) 1939.

[19] J.R. Sanmartín, O. López-Rebollal and N. de Paola, Physica D 69 (1993) 148;

J.R. Sanmartín, in: Synergetics, Order and Chaos, M.G. Velarde, ed. (World Scientific, Singapore, 1988) pp. 55-65.

[20] D.A. Russell and E. Ott, Phys. Fluids 24 (1981) 1976.

[21] J.J. Collins and I. Stewart, Biol. Cybern. 71 (1994) 95.

[22] R.L. Devaney, An Introduction to Chaotic Dynamical Systems (Benjamin/Cummings, Menlo Park, CA, 1986) Section 1.5.

[23] C. Grebogi, E. Ott and J.A. Yorke, Phys. Rev. Lett. 48 (1982) 1507; Physica D 7 (1983) 181. . 